\title{
CRescimento e nutrição Mineral de Ipomoea nil
}

\author{
Growth and Mineral Nutrition of Ipomoea nil Roth
}

\author{
DUARTE, D. J. ${ }^{2}$, BIANCO, S. ${ }^{3}$, MELO, M. N. ${ }^{4}$ e CARVALHO, L.B. ${ }^{5}$
}

\begin{abstract}
RESUMO - Um experimento em casa de vegetação foi conduzido entre novembro de 2005 e abril de 2006, na UNESP, campus de Jaboticabal, SP, objetivando estudar a produção de massa seca, a distribuição e o acúmulo de macronutrientes por Ipomoea nil, uma importante infestante de culturas anuais e perenes no Brasil. As plantas foram cultivadas em vasos de 7 litros com substrato de areia e irrigadas diariamente com solução nutritiva de Hoagland \& Arnon. O delineamento experimental foi inteiramente casualizado, com quatro repetições. Os tratamentos corresponderam às épocas de avaliação, em intervalos de 14 dias, iniciandose 21 dias após a emergência (DAE). Em cada avaliação, as plantas de quatro vasos foram analisadas quanto ao acúmulo de massa seca e à nutrição mineral. I. nil apresentou pequeno acúmulo de massa seca e de macronutrientes no início da fase experimental. Esses acúmulos se intensificaram após $77 \mathrm{DAE}$, atingindo o máximo valor teórico aos 123, 122, 124, 121, 119, 121 e 125 DAE, para massa seca, N, P, K, Ca, Mg e S, respectivamente. Folhas e caules foram os principais órgãos responsáveis pelo acúmulo de massa seca por plantas de I. nil. $\mathrm{K}$ e $\mathrm{N}$ foram os macronutrientes extraídos em maior quantidade por essa espécie.
\end{abstract}

Palavras-chave: plantas daninhas, corda-de-viola, massa seca, macronutrientes.

\begin{abstract}
A greenhouse experiment was carried out from November 2005 through April 2006, at UNESP, Jaboticabal, SP, Brazil, to study dry mass production and macronutrient distribution and accumulation by Ipomoea nil, an important weed of annual and perennial crops in Brazil. The plants were cultivated in 7 liter pots, with sand substrate daily irrigated with Hoagland \& Amon nutrient solution. The experimental design was completely randomized with four replications. The treatments corresponded to the evaluation times, at 14 day intervals, beginning 21 days after emergence (DAE). In each evaluation, the plants from four pots were analyzed for dry mass accumulation and mineral nutrition. I. nil showed small dry mass and macronutrient accumulations at the beginning of the experimental stage. These accumulations intensified after $77 D A E$, reaching the maximum theoretical value at 123, 122, 124, 121, 119, 121 and 125 DAE, for dry mass, $N, P$, $K, \mathrm{Ca}, \mathrm{Mg}$ and $\mathrm{S}$, respectively. Leaves and stems were the main organs responsible for dry mass accumulation by I. nil plants. $K$ and $N$ were the macronutrients extracted at higher amount by this species.
\end{abstract}

Keywords: weeds, morning-glory, dry mass, macronutrients.

1 Recebido para publicação em 2.10.2007 e na forma revisada em 29.5.2008.

Parte do trabalho de graduação do primeiro autor apresentado à FCAV/UNESP - Jaboticabal-SP,

2 Mestrando do Programa de Pós-Graduação em Produção Vegetal da Faculdade de Ciências Agrárias e Veterinárias, Universidade Estadual Paulista - FCAV/UNESP, Jaboticabal-SP, <duarte_dj@yahoo.com.br>; ${ }^{3}$ Professor Livre-Docente do Dep. de Biologia Aplicada à Agropecuária, FCAV/UNESP, Via de Acesso Prof. Paulo Donato Castellane s/n, 14884-900 Jaboticabal-SP; ${ }^{4}$ Acadêmica do curso de Agronomia - FCAV/UNESP, Jaboticabal-SP, ${ }^{5}$ Doutorando do Programa de Pós-Graduação em Produção Vegetal da FCAV/UNESP, Jaboticabal-SP. 


\section{INTRODUÇÃO}

Ipomoea é o gênero que mais se destaca no âmbito da família Convolvulaceae, com 600-700 espécies, no mundo. Dentre as espécies do gênero, existem diversas plantas daninhas que são importantes infestantes de culturas anuais e perenes, destacando-se Ipomoea hederifolia, Ipomoea quamoclit, Ipomoea purpurea, Ipomoea triloba e Ipomoea nil, denominadas cordas-de-viola. No Brasil, I. nil apresenta vasta e expressiva ocorrência, sendo uma das espécies do gênero mais freqüentes nas áreas de produção agrícola (Kissmann \& Groth, 1999). Atualmente, esta espécie tem sido crítica em plantações de cana-de-açúcar, milho, soja e outras culturas, tornando-se uma grande preocupação para os produtores brasileiros.

Considerando a importância atual de $I$. nil como planta infestante, há necessidade de estudos básicos a respeito da biologia dessa espécie, envolvendo aspectos relacionados a reprodução, crescimento, desenvolvimento, exigências em nutrientes, respostas aos sistemas de controle e outros, visando obter informações que possam auxiliar no seu manejo.

De acordo com Pitelli (1983), plantas daninhas, em geral, possuem diversificados e complexos mecanismos de adaptação, que thes permitem, em curto prazo, sobreviver às variações do ambiente e reproduzir-se em condições adversas, graças à sua alta capacidade de alteração de fenótipo, como resposta às variações do meio. Esse fenômeno, denominado plasticidade fenotípica, visa um ajuste ecofisiológico da planta a um ambiente específico.

Além disso, as plantas daninhas podem sofrer modificações evolutivas, que ocorrem em toda a população, sendo necessárias várias gerações para que as modificações sejam expressivas; entretanto, ficam fixadas no patrimônio genético e são repassadas às gerações subseqüentes. Segundo Bianco et al. (2004), estudos básicos de ecofisiologia e das relações de cada espécie de planta daninha são fundamentais para entender seu comportamento sob diferentes condições ambientais e para fornecer subsídios para predições de seu sucesso como infestante em função de novas práticas agrícolas e da introdução em novos ambientes.

Estudos a respeito de requerimentos nutricionais por plantas daninhas são de suma importância para a ciência das plantas daninhas, pelo fato de que, segundo Pitelli (1985), a competição por nutrientes é um dos principais fatores ecológicos que afetam negativamente a produtividade das culturas agrícolas, assim como a competição por luz.

Os trabalhos publicados a respeito de requerimentos nutricionais de plantas daninhas são poucos, podendo-se citar os de Rodrigues et al. (1995), com Commelina benghalensis; Erasmo et al. (2000), com Senna obtusifolia; Souza Filho et al. (2000), com Urena lobata; Gravena et al. (2002), com Hyptis suaveolens; Brighenti et al. (2003), com Cardiospermum halicacabum; Pedrinho Júnior et al. (2004), com Glycine maxe Richardia brasiliensis; Kazda et al. (2004) com Quercus petraea, Fagus sylvatica e Acer pseudoplatanus e Carvalho et al. (2007) com Zea mays e Brachiaria plantaginea, entre outros.

O presente trabalho objetivou estudar a produção de massa seca, a marcha de absorção e o acúmulo de macronutrientes por plantas de $I$. nil, cultivadas em condições padronizadas de nutrição mineral.

\section{MATERIAL E MÉTODOS}

O experimento foi conduzido em casa de vegetação do Departamento de Biologia Aplicada à Agropecuária, da Faculdade de Ciências Agrárias e Veterinárias - UNESP - campus de Jaboticabal, SP, no periodo de novembro de 2005 a abril de 2006, utilizando vasos plásticos com capacidade para 7 litros, tendo como substrato para crescimento areia de rio lavada e peneirada.

A semeadura foi feita em meados de novembro de 2005. Quando as mudas atingiram o estádio de dois pares de folhas verdadeiras totalmente expandidas, foi efetuado o desbaste, deixando-se apenas quatro plantas por vaso. Os vasos foram irrigados com solução nutritiva completa de Hoagland \& Arnon (1950), recebendo $25 \%$ da concentração original durante os primeiros 35 dias e, posteriormente, $50 \%$ até o final da fase experimental. 
O delineamento experimental utilizado foi inteiramente casualizado, com dez tratamentos e quatro repetições. Os tratamentos constituíram-se em épocas de avaliação, realizadas em intervalos de 14 dias: $21,35,49,63,77$, $91,105,119,133$ e 147 dias após a emergência (DAE). As repetições constituíram-se nos quatro vasos avaliados por época de avaliação.

Em cada época de avaliação, as plantas de quatro vasos foram coletadas e separadas em raizes, caules, folhas e estruturas reprodutivas (flores + frutos). A seguir, todo o material foi lavado conforme Sarruge \& Haag (1974), sendo submetido a rápida imersão em solução diluída de detergente neutro, lavagem por imersão em água destilada e, finalmente, imersão em água deionizada. Após lavagem, as diferentes partes das plantas foram acondicionadas em sacos de papel devidamente identificados e perfurados, para posterior secagem em estufa de renovação forçada de ar a 60$70^{\circ} \mathrm{C}$ por 96 horas.

A massa seca foi obtida pesando-se o material seco em balança eletrônica com precisão de centésimos de gramas. Após a pesagem, o material foi moído em micromoinho tipo Willey, em malha $20 \mathrm{~mm}$, e armazenado em frascos de vidros hermeticamente fechados. Após a moagem do material vegetal, foram determinados os teores de macronutrientes.

O nitrogênio total ( $\mathrm{N}_{\text {total }}$ ) e o fósforo (P) foram determinados pelos métodos semimicro kjedahl e colorimétrico do ácido fosfovanadatomolíbdico, respectivamente, conforme descrito por Sarruge \& Haag (1974). Na extração do potássio $(\mathrm{K})$, do cálcio $(\mathrm{Ca})$ e do magnésio $(\mathrm{Mg})$ foi utilizado o método descrito por Jorgensen (1977), por meio de espectrofotometria de absorção atômica. O S foi determinado pelo método turbidimétrico, descrito por Vitti (1989).

Os acúmulos de nutrientes para as partes da planta foram descritos multiplicando-se o teor do nutriente pela massa seca correspondente. O acúmulo total foi obtido por meio da somatória dos acúmulos das diferentes partes da planta, enquanto o teor total da planta foi obtido pela relação entre o acúmulo total da planta e a massa seca total correspondente.

Para cálculo do acúmulo total teórico de massa seca e macronutrientes, utilizou-se a equação exponencial $y=\exp \left(a+b x+c x^{2}\right)$, sendo y o acúmulo de massa seca e macronutriente e $\mathrm{x}$ os dias após a emergência. As curvas foram ajustadas utilizando-se o software Statistica 6.0 (Statsoft, 2006), em função dos dias do ciclo de vida da planta, refletindo um esboço comportamental da espécie em relação ao nutriente estudado.

Os pontos de máximo acúmulo teórico de massa seca e macronutriente foram determinados pela derivada primeira e segunda da equação ajustada aos dados obtidos, utilizando o software Maple V (Chart et al., 1991).

\section{RESULTADOS E DISCUSSÃO}

O acúmulo de massa seca por plantas de I. nil foi pequeno até próximo da metade do ciclo de desenvolvimento da planta e atingiu o valor máximo teórico aos $123 \mathrm{DAE}$, quando essa espécie acumulou $33,56 \mathrm{~g}$ por planta (Figura 1A). Após esse período, houve diminuição no acúmulo de massa seca em virtude da senescência das plantas. Observou-se grande acúmulo de massa seca total no período de 77 a 119 DAE; nesse último período, I. nil acumulou $31,91 \mathrm{~g}$ por planta. Isso ocorreu em função do aumento no acúmulo de massa seca pelas diferentes partes da planta, principalmente dos caules, como pode ser observado na Figura 1B.

Deve-se ressaltar que $I$. nil é uma planta de ciclo de desenvolvimento mais prolongado que o das culturas anuais, podendo causar interferência na colheita, pelo fato de seus ramos emaranharem-se nas plantas cultivadas, ocasionando transtornos e perdas indiretas de produtividade (Kissmann \& Groth, 1999).

No início do ciclo de desenvolvimento de $I$. nil, o percentual de massa seca acumulada em folhas foi maior que aquele acumulado em outras partes da planta (Figura 2). O percentual de massa seca acumulada em raízes dessa espécie esteve em torno de $30 \%$, do início até próximo da metade do ciclo de desenvolvimento da planta, e diminuiu após $77 \mathrm{DAE}$. Isso ocorreu, provavelmente, para que houvesse melhor fixação da planta no substrato, nos períodos iniciais do seu ciclo de desenvolvimento, fazendo com que ocorresse aumento no contato dos nutrientes por interceptação radicular, levando ao rápido acúmulo destes pelas raízes (Carvalho et al., 2007). 


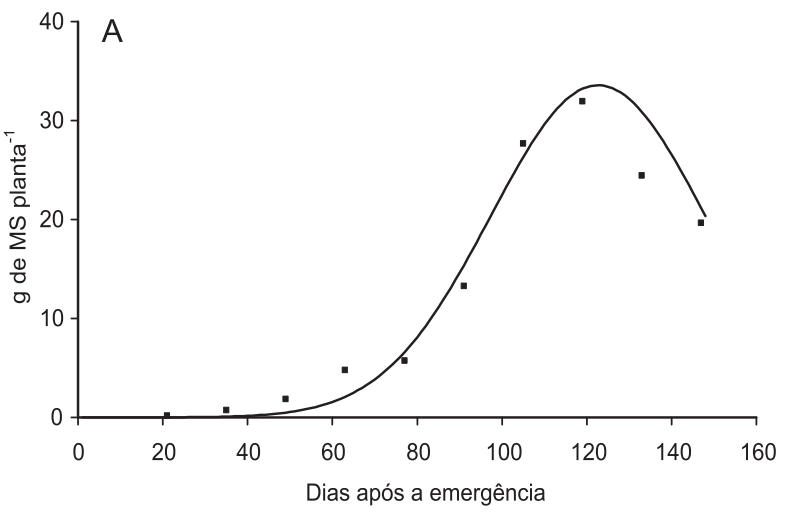

$y=\exp \left((-8,0323)+(0,189796)^{*} x+(-0,78 e-3) \& x^{2}\right.$

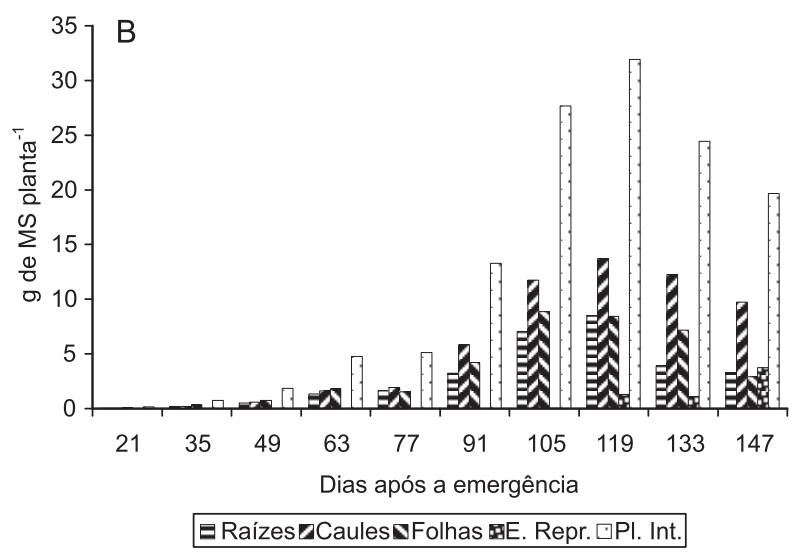

Figura 1 - Curva de regressão da média dos dados de acúmulo de massa seca na planta inteira (A) e evolução do acúmulo de massa seca na planta inteira e nas diferentes estruturas constituintes das plantas de Ipomoea nil (B), ao longo do seu ciclo de desenvolvimento. FCAV/UNESP, JaboticabalSP, 2005

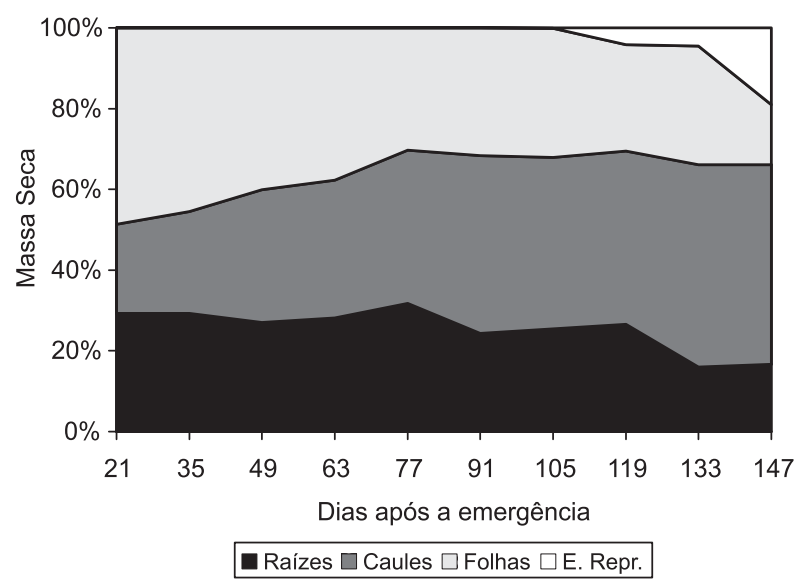

Figura 2 - Partição de massa seca (\%) nas diferentes estruturas constituintes das plantas de Ipomoea nil, ao longo do seu ciclo de desenvolvimento. FCAV/UNESP, Jaboticabal-SP, 2005 .
O percentual de massa seca acumulada em caules aumentou desde os $21 \mathrm{DAE}$, em detrimento das folhas, principalmente, o que proporcionou maior percentual de massa seca acumulada em caules do que em outras partes da planta de 77 a $147 \mathrm{DAE}$. Isso se deve ao hábito de crescimento trepador de I. nil (Kissmann \& Groth, 1999), cujos caules se desenvolvem rapidamente para a sustentação dos ramos das plantas, em busca de luz.

O florescimento de $I$. nil iniciou-se entre 91 e 105 DAE, tendo o maior acúmulo de massa seca nas estruturas reprodutivas ocorrido aos $147 \mathrm{DAE}$, quando o acúmulo foi de $3,73 \mathrm{~g}$ por planta (Figura 1B). O percentual de massa seca acumulada pelas estruturas reprodutivas foi pequeno e atingiu o maior valor (19\%) aos 147 DAE (Figura 2).

É importante ressaltar que houve intensa diminuição no percentual de massa seca acumulada em folhas a partir do início do florescimento, em razão da mudança do dreno principal das folhas para as estruturas reprodutivas. Esse fato também foi observado em outras espécies daninhas, de acordo com relatos de Rodrigues (1992), em C. benghalensis; Pedrinho Júnior (2003), em R. brasiliensis, $H$. suaveolens e Alternanthera tenella; Bianco (2003), em Euphorbia heterophylla, Sida rhombifolia, S. obtusifolia, Desmodium tortuosum e Solanum americanum; Bianco et al. (2004), em Rottboellia exaltata; e Carvalho et al. (2007), em B. plantaginea.

Os macronutrientes com maiores teores médios encontrados nas plantas de $I$. nil foram $\mathrm{K}, \mathrm{N}, \mathrm{Ca}, \mathrm{Mg}, \mathrm{P}$ e S (Tabela 1). Os teores dos macronutrientes nas plantas de $I$. nil oscilaram durante toda a fase experimental. Aos $35 \mathrm{DAE}$ foi observado maior teor de $\mathrm{Mg}$, enquanto aos $63 \mathrm{DAE}$ foi verificado maior teor de $\mathrm{N}, \mathrm{P}$ e $\mathrm{S}$ e, aos $77 \mathrm{DAE}$, maior teor de $\mathrm{K}$ e Ca. De acordo com os resultados dos trabalhos realizados por Bianco (2003) e Pedrinho Júnior (2003), constatou-se que K e N são os macronutrientes que estão presentes em maiores concentrações nas plantas daninhas de "folhas largas" já estudadas, como D. tortuosum, $S$. obtusifolia, $S$. americanum, $H$. suaveolens, S. rhombifolia, E. heterophylla, $R$. brasiliensis e A. tenella.

$\mathrm{O}$ acúmulo total dos macronutrientes foi pequeno no início do ciclo de desenvolvimento 
Tabela 1 - Teor de macronutrientes na planta inteira de Ipomoea nil, ao longo do ciclo de desenvolvimento. FCAV/UNESP, Jaboticabal-SP, 2005

\begin{tabular}{|c|c|c|c|c|c|c|}
\hline \multirow{2}{*}{ DAE } & $\mathrm{N}_{\text {total }}$ & $\mathrm{P}$ & $\mathrm{K}$ & $\mathrm{Ca}$ & $\mathrm{Mg}$ & $\mathrm{S}$ \\
\cline { 2 - 7 } & $------------------\mathrm{g} \mathrm{kg}^{-1}$ de massa seca & ---------------- \\
\hline 21 & 22,99 & 1,99 & 24,54 & 11,54 & 5,18 & 1,65 \\
\hline 35 & 27,84 & 1,81 & 27,67 & 13,77 & 5,40 & 1,42 \\
\hline 49 & 29,55 & 1,51 & 35,69 & 14,10 & 5,12 & 1,49 \\
\hline 63 & 30,54 & 2,43 & 34,08 & 14,51 & 4,81 & 1,84 \\
\hline 77 & 24,71 & 2,40 & 58,29 & 15,30 & 4,63 & 1,73 \\
\hline 91 & 28,04 & 2,04 & 30,61 & 13,10 & 4,44 & 1,77 \\
\hline 105 & 26,31 & 1,61 & 26,38 & 11,57 & 3,88 & 1,33 \\
\hline 119 & 23,99 & 1,45 & 26,24 & 12,05 & 4,12 & 1,06 \\
\hline 133 & 28,94 & 1,86 & 29,00 & 13,54 & 4,61 & 1,69 \\
\hline 147 & 24,93 & 1,72 & 26,70 & 10,30 & 3,82 & 1,50 \\
\hline
\end{tabular}

$\mathrm{DAE}$ - dias após a emergência.

de I. nil (Figura 3). Esse acúmulo foi crescente após o primeiro terço do ciclo, e o máximo acúmulo teórico ocorreu aos 122, 124, 121, 119, 121 e 125 DAE, respectivamente, para N, P, K, $\mathrm{Ca}, \mathrm{Mg}$ e $\mathrm{S}$. Os valores de acúmulo máximo teórico foram de $791,79 \mathrm{mg}$ de $\mathrm{N}_{\text {planta }}{ }^{-1}$, 50,82 mg de $\mathrm{P}$ planta $^{-1}, 780,63 \mathrm{mg}$ de K planta $^{-1}$, $362,96 \mathrm{mg}$ de Ca planta $^{-1}, 139,68 \mathrm{mg}$ de Mg planta $^{-1}$ e 38,71 mg de S planta ${ }^{-1}$, respectivamente para cada período de acúmulo máximo. Observou-se grande acúmulo de N, P e S dos 77 aos 105 DAE (Figura 3A, B, F); nesse último período, $I$. nil acumulou $730,04 \mathrm{mg}$ de $\mathrm{N}$ planta $^{-1}$, 44,63 mg de P planta ${ }^{-1}$ e $36,78 \mathrm{mg}$ de S planta ${ }^{-1}$. Verificou-se, também, grande acúmulo de K, Ca e $\mathrm{Mg}$ dos 77 aos $119 \mathrm{DAE}$ (Figura 3C, D, E); nesse último período, I. nil acumulou 727,94 mg de K planta ${ }^{-1}, 320,26 \mathrm{mg}$ de Ca planta ${ }^{-1}$ e 107,40 mg de Mg planta ${ }^{-1}$.

Ao comparar os resultados obtidos neste trabalho com aqueles envolvendo outras espécies de plantas daninhas de "folhas largas" já estudadas, até o ponto de acúmulo máximo teórico considerado para cada macronutriente, pôde-se constatar que $I$. nil apresentou acúmulo máximo teórico para $\mathrm{N}, \mathrm{K}, \mathrm{Mg}$ e $\mathrm{S}$ menor que os observados para $D$. tortuosum, S. obtusifolia, S. americanum (Bianco, 2003) e H. suaveolens (Pedrinho Júnior, 2003), porém maiores que os observados para $S$. rhombifolia, E. heterophylla (Bianco, 2003), R. brasiliensis e A. tenella (Pedrinho Júnior, 2003). Quanto aos acúmulos de $\mathrm{P} \mathrm{e} \mathrm{Ca,} \mathrm{os} \mathrm{valores} \mathrm{foram} \mathrm{me-}$ nores que os observados para todas as espécies supracitadas, indicando assim que $I$. nil é uma planta daninha menos exigente em termos nutricionais que $D$. tortuosum, $S$. obtusifolia, $S$. americanum e $H$. suaveolens.

Os macronutrientes acumulados em maiores quantidades foram justamente aqueles encontrados em maiores concentrações nas plantas de I. nil; assim, a ordem de extração foi $\mathrm{K}>\mathrm{N}>\mathrm{Ca}>\mathrm{Mg}>\mathrm{P}>\mathrm{S}$. Com base nos resultados dos trabalhos realizados por Bianco (2003) e Pedrinho Júnior (2003), constatou-se que $\mathrm{K}$ e $\mathrm{N}$ são os macronutrientes mais acumulados pelas plantas daninhas de "folhas largas" já estudadas. Sabe-se, também, que esses dois elementos são os macronutrientes exigidos em maior quantidade pela maioria das culturas de interesse econômico (Malavolta et al., 1997). Portanto, a competição por nutrientes imposta pelas plantas de $I$. nilé um fator biótico muito importante, que pode afetar negativamente o desenvolvimento e a produtividade das plantas cultivadas.

Considerando o período de maior competição das plantas daninhas com a maioria das culturas anuais como sendo por volta de $77 \mathrm{DAE}$, época em que se intensificou a extração dos macronutrientes estudados, uma planta de $I$. nil acumulou nesse período 5,73 gramas de massa seca; 136,72 mg de N; 13,29 mg de P; 322,86 mg de K; 85, 13 mg de Ca; 25,74 mg de Mg; e 9,53 mg de S.

Folhas e caules são, portanto, os principais órgãos responsáveis pelo acúmulo de massa seca por plantas de $I$. nil, na primeira e na segunda metade do ciclo de desenvolvimento dessa espécie, respectivamente. $\mathrm{K}$ e $\mathrm{N}$ são os macronutrientes extraídos em maior quantidade pelas plantas de $I$. nil. O período de máximo acúmulo teórico de massa seca e macronutrientes ocorreu entre 119 e 125 dias após a emergência das plantas de $I$. nil. 

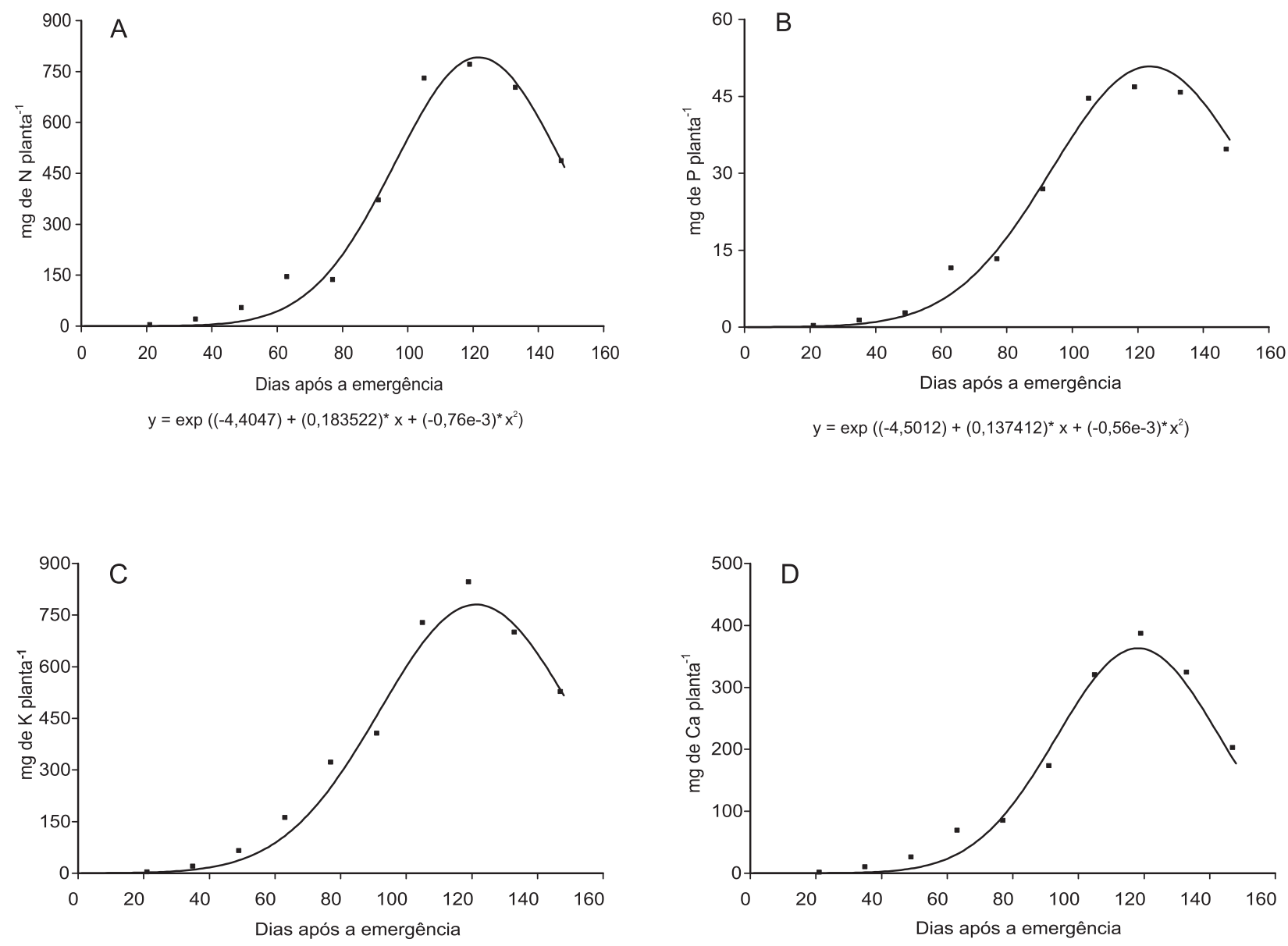

$y=\exp \left((-1,7387)+(0,13959)^{*} x+(-0,58 e-3)^{*} x^{2}\right)$

$y=\exp \left((-5,2317)+(0,189868)^{*} x+(-0,81 e-3)^{*} x^{2}\right)$


Figura 3 - Curva de regressão da média dos dados de acúmulo de nitrogênio (A), fósforo (B), potássio (C), cálcio (D), magnésio (E) e enxofre (F), na planta inteira de Ipomoea nil, ao longo do seu ciclo de desenvolvimento. FCAV/UNESP, Jaboticabal-SP, 2005. 


\section{LITERATURA CITADA}

BIANCO, S. Estudo comparativo do acúmulo de massa seca e de macronutrientes por plantas de soja cv. BR-16 e cinco espécies daninhas. 2003. 95 f. Tese (Livre Docência) - Universidade Estadual Paulista, Jaboticabal, 2003.

BIANCO, S.; BARBOSA JÚNIOR, A. F.; PITELLI, R. A. Crescimento e nutrição mineral de capim-camalote. Planta Daninha, v. 22, n. 3, p. 375-380, 2004

BRIGHENTI, A. M.; VOLL, E.; GAZZIERO, D. L. P. Biologia e manejo do Cardiospermum halicacabum. Planta Daninha, v. 21, n. 2, p. 229-237, 2003.

CARVALHO, L. B. et al. Estudo comparativo do acúmulo de massa seca e macronutrientes por plantas de milho var. BR106 e Brachiaria plantaginea. Planta Daninha, v. 25, n. 2, p. 293-301, 2007.

CHART, B. W. et al. Maple V: language reference manual New York: Springer-Verlag, 1991. 411 p.

ERASMO, E. A. L. et al. Efeito de níveis crescentes de calagem no crescimento e estado nutricional de fedegoso Planta Daninha, v. 18, n. 2, p. 253-263, 2000

GRAVENA, R. et al. Análise do crescimento de Hyptis suaveolens. Planta Daninha, v. 20, n. 2, p. 189-196, 2002

HOAGLAND, D. R.; ARNON, D. J. The water culture method of growing plants without soil. Berkeley: University of California, 1950. 31 p. (Circular, 347)

JORGENSEN, S. S. Metodologia utilizada para análises químicas de rotina: guia analítico. Piracicaba: CENA, 1977. $24 \mathrm{p}$.

KAZDA, M. et al. Importance of mineral nutrition for photosynthesis and growth of Quercus petraea, Fagus sylvatica and Acer pseudoplatanus planted under Norway spruce canopy. Plant Soil, v. 264, n. 1-2, p. 25-34, 2004

KISSMANN, K. G; GROTH, D. Plantas infestantes e nocivas. 2.ed. São Paulo: BASF, 1999. Tomo II. 978 p.

MALAVOLTA, E.; VITTI, G. C.; OLIVEIRA, S. A Avaliação do estado nutricional das plantas: princípios e aplicações. 2.ed. São Paulo: Potafos, 1997. 319 p.
PEDRINHO JÚNIOR, A. F. F. Estudo comparativo do acúmulo de massa seca e de macronutrientes por plantas de soja cv. BR-16 e três espécies daninhas. 2003. 67 f. Dissertação (Mestrado em Agronomia) - Universidade Estadual Paulista, Faculdade de Jaboticabal, 2003.

PEDRINHO JÚNIOR, A. A. F.; BIANCO, S.; PITELLI, R. A. Acúmulo de massa seca e macronutrientes por plantas de Glycine max e Richardia brasiliensis. Planta Daninha, v. 22, n. 1, p. $53-61,2004$

PITELLI, R. A. Biologia de plantas daninhas. In: DOWER NETO, J.B. - SEMANA DO HERBICIDA, 5., 1983, Bandeirantes: Semana... Fundação Faculdade de Agronomia "Luiz Meneghel", 1983. p. 1-9. (Apostila).

PITELLI, R. A. Interferência de plantas daninhas em culturas agrícolas. Inf. Agropec., v. 11, n. 129, p. 16-27, 1985.

RODRIGUES, B. N. Estudos sobre a dormência, absorção de macronutrientes e respostas à calagem por Commelina benghalensis L., 1992. 129 f. Tese (Doutorado em Agronomia) - Universidade Estadual Paulista, Jaboticabal, 1992

RODRIGUES, B. N.; PITELLI, R. A.; BELLINGIERI, P. A Efeitos da calagem do solo no crescimento inicial e absorção de macronutrientes por plantas de trapoeraba (Commelina benghalensis). Planta Daninha, v. 13, n. 2, p. 59-68, 1995.

SARRUGE, J. R.; HAAG, H. P. Análises químicas em plantas. Piracicaba: Escola Superior de Agricultura Luiz de Queiroz, 1974. 56 p.

SOUZA FILHO, A. P. S.; VELOSO, C. A. C.; GAMA, J. R. N. Capacidade de absorção de nutrientes do capim-marandu (Brachiaria brizantha) e da planta daninha malva (Urena lobata) em função do pH. Planta Daninha, v. 18, n. 3, p. $443-450,2000$

STATSOFT. Data analysis software system. Version 6.0. Disponível em: $<$ http://www.statsoft.com $>$. Acesso em: 16 jul. 2006.

VITTI, G. C. Avaliação e interpretação do enxofre no solo e na planta. Jaboticabal: FUNEP, 1989. 37 p. 